\title{
Confiabilidad y validez de la Escala de Déficit Atencional (EDAH) adaptada en estudiantes chilenos
}

\author{
Marta Belmar Mellado, ' Francisco Pablo Holgado Tello, ${ }^{2}$ Leandro Navas Martínez, ${ }^{3}$ Benjamín Vicente Parada ${ }^{4}$
}

Artículo original

\section{ABSTRACT}

\section{Background}

The needs of children's mental health care make it necessary to have epidemiological studies that serve as a basis to refine the interventions and enhance the diagnosis with reliable and appropriate tools.

\section{Objective}

The objective of this study was to analyze the psychometric properties of the scale for the assessment of the Attentional Deficit Disorder (ADHD) in primary and secondary schools in Chile.

\section{Method}

A sample of 508 students (144 with Attentional deficits, 110 with suspected autism and 254 common) was assessed through a test completed by their teachers.

\section{Results}

The factorial structure of the ADHD revealed that three factors explain together the $71.61 \%$ of the total variance of the scale, with a coefficient alpha of 0,948 . This allows us to conclude that the scale presents acceptable psychometric characteristics and adequate internal consistency, and that its elements have a suitable discriminatory power.

\section{Discussion and conclusion}

The present study of mental health in Chilean schoolchildren revealed that the ADHD adapted scale possesses a high discriminant capacity and adequate elements and factors reliability, as well as an optimal discriminant power among factors. We analyze the implications and possible contribution to the early detection of clinical diagnosis in mental health.

Key words: Instrument validity, reliability, attention deficit disorders with hyperactivity.

\section{RESUMEN}

\section{Antecedentes}

Las necesidades de atención en salud mental infantil hacen necesario contar con estudios epidemiológicos que sirvan de base para perfeccionar las intervenciones y acrecentar el diagnóstico, con instrumentos fiables y adecuados al contexto.

\section{Objetivo}

El objetivo de este estudio fue analizar las propiedades psicométricas de la Escala para la Evaluación del Trastorno por Déficit Atencional (EDAH), en escolares de primaria y secundaria de Chile.

\section{Método}

Se evaluó una muestra de 508 alumnos (144 con Déficit Atencional, 110 con sospecha de padecerlo y 254 comunes) mediante un test completado por sus profesores.

\section{Resultados}

La estructura factorial de la EDAH reveló tres factores que en conjunto explican el $71,61 \%$ de la varianza total de la escala, con un coeficiente Alfa de 0,948. Esto permite concluir que la adaptación de la escala presenta características psicométricas aceptables, una adecuada consistencia interna y que sus elementos poseen un adecuado poder discriminatorio.

\section{Discusión y conclusión}

Se efectuó el estudio transversal de salud mental en escolares chilenos, el cual reveló que la escala EDAH adaptada posee alta capacidad de discriminación, adecuada fiabilidad de elementos y factores, además de presentar un óptimo poder discriminante entre factores. Se analizan las implicaciones y el posible aporte en la detección precoz del diagnóstico clínico en salud mental.

Palabras clave: Validez de instrumentos; confiabilidad; trastornos de la atención con hiperactividad.

1 Facultad de Educación, Departamento de Fundamentos de la Educación, Universidad Católica del Maule, Chile.

2 Facultad de Psicología, Departamento de Metodología de las Ciencias del Comportamiento. Universidad Nacional de Educación a Distancia (UNED), España.

3 Facultad de Educación, Departamento de Psicología Evolutiva y Didáctica. Universidad de Alicante, España.

4 Facultad de Medicina, Departamento de Psiquiatría y Salud Mental. Universidad de Concepción, Chile.

Correspondencia: Leandro Navas Martínez. Departamento de Psicología Evolutiva y Didáctica. Apartado Correos, 99. 03080 Alicante, España. Tel: (34-96) 590-3400 Ext. 2913. Fax: (34-96) 590-3495. E-mail: leandro.navas@ua.es

Recibido primera versión: 27 de enero de 2014. Segunda versión: 27 de noviembre de 2014. Aceptado: 28 de enero de 2015 


\section{ANTECEDENTES}

En la actualidad, el trastorno por déficit atencional con hiperactividad (TDAH) es uno de los trastornos infantiles más diagnosticados y tratados en la clínica infanto-juvenil. Existen discrepancias frente a las cifras reales, debido a la diversidad de criterios diagnósticos o bien porque los instrumentos utilizados varían tanto en la metodología como en las fuentes de información. Pese a los desacuerdos, la prevalencia oscila entre 3 y $7 \%{ }^{1}$ o entre el 1 y $2 \%,{ }^{2}$ aunque a nivel global se sitúan las tasas entre 8 y 12\%. ${ }^{3}$ Las cifras españolas no se alejan de las internacionales, ya que estudios revelan la presencia del trastorno entre 3 y 5\%, ${ }^{4}$ mientras que en Chile las tasas de prevalencia revelan un $6.2 \%$ en los estudiantes. ${ }^{5}$

Si bien los estudios epidemiológicos de salud mental en escolares han sido insuficientes, hay antecedentes que dan cuenta de la prevalencia entre seis y 11 años en Santiago de Chile, ${ }^{6}$ donde se reporta que $7.2 \%$ obtenía puntajes altos para problemas conductuales sin diferencias de género y que variaba inversamente al nivel socioeconómico. Los problemas conductuales se presentaron en $8.4 \%$ de los niños de 10 y 11 años comparado con el 6.5\% de los menores de 10 años. $^{7}$

Un estudio de prevalencia de trastornos psiquiátricos en la población escolar de primer año básico, en Santiago de Chile, ${ }^{8}$ observó que más de $24 \%$ de escolares presentaba algún tipo de trastorno, ${ }^{5}$ que un $47 \%$ no cumplía con criterios para un diagnóstico psiquiátrico, y que sólo el 13\% de los niños con la patología recibieron atención especializada. ${ }^{9}$ Sin embargo, existieron discrepancias con respecto al tipo de intervención. ${ }^{10}$

Otra investigación con niños y adolescentes chilenos (cuatro a 11 años y de 12 a 18 años) en la que se utilizó la versión computarizada de la entrevista DISC-IV, reveló que el trastorno disruptivo de mayor prevalencia en el país fue el TDAH y que las diferencias de género no fueron significativas. Las prevalencias más bajas no se presentaron en Santiago, sino en Concepción y Cautín. Por otra parte, Santiago y Cautín mostraron mayor prevalencia en niños que en adolescentes. El estudio mostró que si bien los trastornos disruptivos son más altos que la mayoría de los estudios en otros países con entrevistas psiquiátricas, la prevalencia igual en hombres que en mujeres del TDAH es distinta a la mayoría de las cifras de otros países. ${ }^{11}$ Las mujeres presentaron prevalencias mayores que los hombres, lo que difiere de lo habitualmente reportado, pero coincide con algunos estudios latinoamericanos recientes. ${ }^{12}$

Si bien los estudios locales o internacionales han reportado elevadas tasas, la mayoría de los autores estima que la prevalencia global de los TDAH en sus diversas formas oscila entre un 3 y 7\%, lo que coincide con las cifras del DSMIV.${ }^{13}$ Esta variabilidad en las prevalencias puede ser causada por las diferencias en los términos, en las definiciones del trastorno, en los puntos de corte y en los criterios diagnósticos, así como en la selección de las muestras. ${ }^{14}$

El TDAH ocasiona problemas en el control ejecutivo del comportamiento, afecta a procesos y funciones ejecutivas implicadas en tareas de enseñanza-aprendizaje, y en la adaptación familiar, escolar y social. Puede presentarse durante los 12 primeros años de vida ${ }^{15}$ y se identifica por falta de atención, impulsividad, intolerancia a la frustración, hiperactividad, dificultades en la atención sostenida y la presencia de trastornos del aprendizaje. ${ }^{16}$ La realidad chilena indica que la población logra ser diagnosticada a partir de la incorporación al sistema educativo y, sobre todo, en la enseñanza básica por ser en esta etapa donde mayormente se evidencian algunas dificultades de aprendizaje, atención y concentración, así como alteraciones de conducta e impulsividad. Estas dificultades requieren una detallada evaluación de los aspectos educativos ${ }^{17}$ y llegan a ser un problema de salud pública relevante, ya que su impacto sobre el niño, la familia, la escuela y la sociedad es altamente significativo y de consecuencia a largo plazo. ${ }^{5}$

La sintomatología de gran impacto en el desarrollo del individuo interfiere en su funcionamiento socio-emocional y cognitivo. Causa, además, una importante disfuncionalidad a nivel personal, grupal y familiar, por lo que se requiere no sólo detectar precozmente los síntomas, sino también intervenir oportunamente. Para favorecer el desarrollo psicológico normal, prever la cronificación y la continuidad de comorbilidades en la etapa adulta.

Sin embargo, una de las dificultades presentes en la salud pública chilena es no contar con instrumentos adecuados que permitan identificar tempranamente el trastorno, o bien que los existentes no recogen en su totalidad las manifestaciones del cuadro. Por lo tanto, aun cuando la sintomatología interfiere en la actividad social, académica o laboral propia del nivel de desarrollo de los sujetos, no se dispone de herramientas clínicas estandarizadas para evaluar el grado de disfuncionalidad. Esta circunstancia hace habitual el que se les etiquete de malos estudiantes ya que, académicamente, sus calificaciones suelen ser bajas, se les suspende más que a sus pares y presentan mayores problemas de aprendizaje, sobre todo cuando no reciben los apoyos oportunos. ${ }^{18}$

Por otra parte, los clínicos precisan la información para mejorar la anamnesis, para definir el cuadro clínico y para juzgar el efecto que los síntomas del TDAH tienen en el rendimiento académico, en las relaciones sociales, escolares y familiares, en la autoestima y en las actividades de la vida diaria. Sin embargo, pese a que la familia y el profesorado colaboran en esta tarea, no siempre es oportuna la recogida de la información y mucho menos la devolución a los centros educativos, lo que entorpece la acción psicopedagógica especializada. Por tanto, para llegar a establecer diagnósticos eficaces y oportunos, se requiere no sólo que los profesores proporcionen la información necesaria e imprescindible para 
el diagnóstico, sino también disponer de instrumentos que permitan recoger las valoraciones de la conducta habitual del alumno, ${ }^{19}$ los cuales deben ser sensibles a las variaciones asociadas a la fuente de información, edad y sexo de la persona evaluada. ${ }^{20}$ Contar con adecuados instrumentos y estudios epidemiológicos permitiría estudiar las características referentes a inatención, impulsividad y trastornos de conducta en la población escolar chilena, lo que facilitaría la acción psicopedagógica diferenciada por género y/o nivel educativo.

\section{MÉTODO Diseño del estudio}

El estudio fue de tipo descriptivo, cuantitativo y no experimental. Se realizó en las provincias de Concepción y Los Ángeles, en la región del Biobío de Chile.

\section{Muestra}

Participaron 219 profesores de enseñanza primaria y secundaria, insertos en establecimientos educacionales públicos y subvencionados de las provincias de Concepción (66.04\%) y de Los Ángeles (35.15\%). El criterio de selección fue que se tratara de docentes del alumnado a evaluar. Todos ellos tuvieron que valorar, con el instrumento que se describirá a continuación, a un total de 508 estudiantes, de los cuales 144 tenían diagnóstico de TDAH; 110 no presentaban un diagnóstico objetivo pero se sospechaba que podían presentarlo y los 254 restantes eran alumnos comunes (no tenían diagnóstico ni se sospechaba que lo pudieran tener) cuadro 1.

Los alumnos del primer grupo ( $\mathrm{n=144)}, 114$ hombres y 30 mujeres, estaban diagnosticados con TDAH por el Servicio de Psiquiatría Infanto-juvenil del Hospital Guillermo Grant Benavente de Concepción y sus edades fluctuaban entre los seis y los 18 años (M=11.47; $\mathrm{DE}=2.98)$. El segundo grupo de alumnos ( $n=110), 67$ hombres y 43 mujeres, no recibía tratamiento en el sector público de salud ni tenía diagnóstico de TDAH, pero durante el semestre habían demandado mucha atención psicopedagógica por presentar problemas de atención-concentración, impulsividad y desajustes conductuales. Es decir, se sospechaba que los alumnos podrían presentar el trastorno. Sus edades fluctuaban entre seis y 14 años $(M=9.76$; $D E=2.07)$. Un tercer grupo $(n=254)$ lo componían alumnos que no presentaban diagnóstico objetivado

Cuadro 1. Distribución de frecuencias por tipo de alumnos

\begin{tabular}{lcccc}
\hline Tipo de alumno & $\mathrm{n}$ & $\mathrm{P}$ & $\mathrm{M}$ & $\mathrm{DE}$ \\
\hline Alumnos con TDAH & 144 & $28.30 \%$ & 11.47 & 2.98 \\
Alumnos con Sospecha & 110 & $21.65 \%$ & 9.76 & 2.07 \\
de TDAH & & & & \\
Alumnos Comunes & 254 & $50.00 \%$ & 10.72 & 2.75 \\
\hline
\end{tabular}

del trastorno ni se sospechaba que lo padecieran. Sus edades variaban entre 6 y 18 años $(M=10.72 ; \mathrm{DE}=2.75)$.

La selección de los alumnos del primer grupo se realizó intencionalmente entre la población consultante del Servicio de Psiquiatría Infanto-Juvenil del Hospital Regional de Concepción. Los alumnos del segundo grupo se seleccionaron de un centro educacional subvencionado incidentalmente en función de no tener diagnóstico clínico pero cuyos profesores sospechaban que podrían padecer TDAH. Los estudiantes del tercer grupo se seleccionaron de los establecimientos educativos que no poseían alumnos en tratamiento en el sector público o privado de salud y por la inexistencia de la patología o de sospecha de la misma. Se tuvo en cuenta la equivalencia de los grupos en el tipo de establecimiento educativo (público o subvencionado), la edad, el nivel educativo y el sexo para que quedaran equiparados en tales variables con los sujetos de los dos primeros grupos.

Los alumnos valorados ( $\mathrm{n}=508), 362$ hombres y $146 \mathrm{mu}$ jeres, presentaron edades entre los seis y 18 años $(M=10.73$; $\mathrm{DE}=2.75)$. El 55.1\% cursaba la enseñanza primaria en centros subvencionados y un $33.9 \%$ en centros públicos. El $5.5 \%$ estudiaba en la secundaria pública y un $4.3 \%$, en centros subvencionados. El 1.2\% restante asistía a centros de educación especial. La mayoría de los alumnos vivía en la comuna de Los Ángeles (254 alumnos), seguida por la comuna de Concepción (162 alumnos), mientras que la minoría habitaba en otras localidades.

\section{Instrumentos y variables}

La Escala para la evaluación del trastorno por déficit de Atención con hiperactividad (EDAH) ${ }^{20}$ permite evaluar los rasgos principales del TDAH de manera sencilla y objetiva por medio de la información aportada por el profesor del sujeto en estudio. ${ }^{20}$ Consta de 20 ítems, con dos subescalas de diez ítems cada una: hiperactividad/déficit de atención y trastornos de conducta. La primera subescala se compone a su vez de dos subescalas, con cinco ítems cada una: hiperactividad/impulsividad y déficit de atención. Las respuestas son valoradas en una escala de tipo Likert de cuatro grados que van desde "Nada" a "Mucha" presencia de la conducta. El marco teórico en el que se inspira es doble. Por una lado, las investigaciones de Conners y las adaptaciones de sus escalas realizadas por los autores y, por el otro, la distinción entre TDAH predominantemente atencional, TDAH predominantemente hiperactivo impulsivo y TDAH combinado que se plantea en el DSM-IV. ${ }^{20}$

Cuadro 2. Coeficientes de fiabilidad de la EDAH original

\begin{tabular}{lccccc}
\hline & $\begin{array}{c}\text { Hiperactividad } \\
\text { impulsividad }\end{array}$ & $\begin{array}{c}\text { Déficit de } \\
\text { atención }\end{array}$ & HDA & $\begin{array}{c}\text { Trastorno } \\
\text { de conducta }\end{array}$ & $\begin{array}{c}\text { Escala } \\
\text { global }\end{array}$ \\
\hline ítems & 5 & 5 & 10 & 10 & 20 \\
Alpha & 0.849 & 0.898 & 0.874 & 0.899 & 0.929 \\
\hline
\end{tabular}


Cuadro 3. Variables de la Escala (Farré y Narbona, 2000)

Variables de la escala con los ítems que la componen

Área I: hiperactividad/impulsividad/inatención

Hiperactividad/Impulsividad: 1, 3, 5, 13 y 17

Déficit de atención: 2, 4, 7, 8 y 19

Área II: trastornos de conducta

$6,9,10,11,12,14,15,16,18$ y 20

La fiabilidad obtenida por los autores, se muestra en el cuadro 2, en tanto que en el cuadro 3 se muestran los ítems que componen la EDAH original, distribuidos por área y factor.

Teniendo presente que la escala EDAH fue construida y baremada en España, para adaptarla al contexto educativo chileno, partiendo del marco teórico de la escala original, se solicitó a un grupo de diez expertos (dos psiquiatras infantiles, tres psicólogos clínicos, dos profesores de enseñanza básica y tres psicopedagogos) que leyesen cada uno de los ítems de la escala original y que formulasen las adecuaciones semánticas necesarias para mejorar la comprensión de los maestros en Chile. ${ }^{21}$ Producto de los ajustes semánticos, todos los ítems de la escala original fueron modificados, lo cual llegó a variar el sentido en que inicialmente estaban redactados. Para efectos de corrección, y a fin de que los ítems no sancionasen la conducta positiva, se invirtió el puntaje original propuesto por los autores en los ítems 6 "Disfruta trabajando en grupo y respeta la opinión de otros", 7 "Habitualmente atiende al profesor mostrando interés por las actividades escolares", 9 "Tiene buenas relaciones interpersonales con sus compañeros", 10 "Reconoce sus errores", 11 "Se comporta adecuadamente en situaciones sociales o de aula", 12 "Admite la autoridad del profesor escuchando sus consejos", 14 "Respeta la opinión y participación de sus compañeros", 16 "El alumno(a) cumple las reglas preestablecidas" y 18 "Es agradable con la mayoría de sus compañeros". Como los cambios implicaban plantear los enunciados en sentido positivo, el puntaje de la escala de evaluación se invirtió a "Nada" con 3 puntos y "Mucho" con 0. Los cambios comentados hacen que algunos elementos se refieran a comportamiento social y de aceptación por parte del grupo.

\section{Procedimiento}

La recogida de datos se obtuvo con el cuestionario recién descrito, que fue administrado a los profesores, quienes fueron informados del objetivo y las características generales del estudio. Se les invitó a completar la escala a partir de la conducta observada en el aula (de los alumnos diagnosticados previamente con el trastorno, de los que se sospechaba trastorno y de los alumnos comunes) y se garantizó la privacidad de la información. Tras obtener el consentimiento verbal, y siguiendo los criterios relativos al consentimiento informado de la Comisión Nacional de Investigación Científica y Tecnológica de Chile, los participantes tuvieron tiempo indefinido para completar la escala, contaron con la asistencia y/o el teléfono de un investigador para aclarar sus dudas y no recibieron retribución.

Paralelamente a la consulta a expertos, se examinaron las fichas clínicas de la población consultante del Servicio de Psiquiatría Infanto-Juvenil del Hospital Regional de Concepción durante el primer semestre del año académico. De los sujetos atendidos ( $n=504)$, el 40.47\% ( $=204)$ estaba diagnosticado con TDAH. Se visitaron sus centros educativos para que los maestros completaran la escala, con lo cual se logró recopilar información de 144 alumnos. Incidentalmente se seleccionó un establecimiento educacional subvencionado de la comuna de Concepción (grupo con sospecha) y el muestreo intencional (grupo común) se realizó con alumnos de centros públicos y subvencionados de la comuna de Los Ángeles.

\section{Análisis estadístico}

El diseño integra un método correlacional con un diseño ex post facto retrospectivo. Se llevaron a cabo análisis descriptivos con el fin de estudiar la dimensionalidad de la escala EDAH (adaptada); análisis factoriales (exploratorio y confirmatorio), análisis multigrupo y análisis de fiabilidad y discriminación. Los procedimientos estadísticos se efectuaron con el software SPSS 20.0 y LISREL 8.71.

\section{RESULTADOS}

De acuerdo con la estructura original propuesta por los autores de la escala, ${ }^{20}$ tal y como se ha señalado en apartados anteriores, la dimensión hiperactividad/impulsividad estaba compuesta por los ítems 1, 3, 5, 13 y 17, la dimensión déficit de atención estaba integrada por los elementos 2, 4, 7, 8 y 19 y, finalmente, la dimensión trastornos de conducta la configuraban los ítems 6, 9, 10, 11,12, 14, 15, 16, 18 y 20. Con estas referencias y teniendo en cuenta, por una parte, las modificaciones semánticas introducidas para adecuarse a la realidad chilena y, por otra parte, las diferencias culturales o sociales que pudiesen existir entre los contextos español y chileno, fue necesario corroborar si tales dimensiones se mantenían. Para responder a esta interrogante, y considerando las respuestas emitidas por los profesores para los sujetos ( $n=508)$, el modelo fue sometido a un Análisis Factorial Confirmatorio (AFC) usando el método de mínimos cuadrados no ponderados. Los índices globales de bondad de ajuste y la solución estandarizada se muestran en el cuadro 4. Dado el elevado error cuadrático, se decide analizar la dimensionalidad de dicho instrumento mediante el Análisis Factorial Exploratorio (AFE) y, posteriormente, con el Análisis Factorial Confirmatorio (AFC). 
Cuadro 4. Solución completamente estandarizada para el modelo teórico

\begin{tabular}{lccc}
\hline Items & $\begin{array}{c}\text { Trastornos } \\
\text { de conducta }\end{array}$ & $\begin{array}{c}\text { Hiperactividad } \\
\text { impulsividad }\end{array}$ & $\begin{array}{c}\text { Déficit } \\
\text { de atención }\end{array}$ \\
\hline 6 & 0.79 & - & - \\
9 & 0.63 & - & - \\
10 & 0.79 & - & - \\
11 & 0.86 & - & - \\
12 & 0.90 & - & - \\
14 & 0.85 & - & - \\
15 & 0.75 & - & - \\
16 & 0.93 & - & - \\
18 & 0.75 & - & - \\
20 & 0.80 & - & - \\
1 & - & 0.73 & - \\
3 & - & 0.89 & - \\
5 & - & 0.72 & - \\
13 & - & 0.84 & - \\
17 & - & 0.90 & - \\
2 & - & - & 0.58 \\
4 & - & - & 0.74 \\
7 & - & - & 0.78 \\
8 & - & - & 0.67 \\
19 & - & - & 0.66 \\
F1 & 1.00 & - & - \\
F2 & 0.80 & 0.78 & - \\
F3 & 0.91 & & 1.00 \\
\hline
\end{tabular}

El AFE se realizó con rotación Varimax y el método de estimación de mínimos cuadrados ponderados. De acuerdo con la estructura original, el gráfico de sedimentación ${ }^{21}$ y el criterio de Kaiser ${ }^{22}$ se extrajeron tres factores y, posteriormente, se analizaron las saturaciones de cada uno de los elementos en el factor correspondiente. La medida de adecuación muestral KMO fue de 0.955 y la prueba de esfericidad de Barlett mostró un $\left.\chi^{2}=8640.46 ; \mathrm{gl}=190 ; \mathrm{p}=0.00\right)$. Se obtuvo la estructura factorial que se muestra en el cuadro 5 .

Las evidencias de validez de constructo aportadas por los análisis exploratorios fueron la base para estudiar la estructura confirmatoria de la EDAH (adaptada). El AFC se realizó por el método de estimación de mínimos cuadrados no ponderados. La especificación del modelo, además de los criterios estadísticos derivados del AFE, siguió los criterios teóricos procedentes de Farré y Narbona. ${ }^{20,23}$ Básicamente, la estructura analizada se correspondió con la obtenida en el AFE. La solución completamente estandarizada, las correlaciones entre las dimensiones del modelo propuesto (modelo 1) y los índices globales de bondad de ajuste se muestran en el cuadro 6 .

A tenor de estos resultados, no sería del todo razonable aceptar la hipótesis nula sobre la adecuación del modelo a los datos (elevado valor del RMSEA). Sin embargo, dado el primer autovalor obtenido en el AFE, así como la alta correlación mostrada entre los tres factores de primer orden, pareció indicado probar una estructura de orden superior.
Cuadro 5. Matriz factorial rotada para las respuestas de la EDAH (adaptada)

\begin{tabular}{|c|c|c|c|c|}
\hline & & $\mathrm{F} 1$ & F2 & F3 \\
\hline 7. & $\begin{array}{l}\text { Habitualmente atiende al profesor } \\
\text { mostrando interés por las actividades } \\
\text { escolares }\end{array}$ & 0.76 & - & - \\
\hline 6. & $\begin{array}{l}\text { Disfruta trabajando en grupo y respe- } \\
\text { ta la participación de otros }\end{array}$ & 0.71 & - & - \\
\hline 11. & $\begin{array}{l}\text { Se comporta adecuadamente en } \\
\text { situaciones sociales o de aula }\end{array}$ & 0.66 & - & - \\
\hline 12. & $\begin{array}{l}\text { Admite la autoridad del profesor, } \\
\text { escuchando sus consejos }\end{array}$ & 0.66 & - & 0.50 \\
\hline 18. & $\begin{array}{l}\text { Es agradable con la mayoría de sus } \\
\text { compañeros }\end{array}$ & 0.65 & - & 0.53 \\
\hline 16. & $\begin{array}{l}\text { El alumno(a) cumple las reglas pre } \\
\text { establecidas }\end{array}$ & 0.64 & 0.42 & - \\
\hline 14. & $\begin{array}{l}\text { Respeta la opinión y participación de } \\
\text { sus compañeros }\end{array}$ & 0.64 & - & 0.45 \\
\hline 2. & Presenta bajo rendimiento escolar & 0.61 & - & - \\
\hline 9. & $\begin{array}{l}\text { Tiene buenas relaciones interpersona- } \\
\text { les con sus compañeros }\end{array}$ & 0.61 & - & 0.49 \\
\hline 10. & Reconoce sus errores & 0.53 & - & 0.49 \\
\hline 8. & No termina las actividades que inicia & 0.51 & 0.42 & - \\
\hline 13. & Necesita moverse constantemente & - & 0.80 & - \\
\hline 1. & $\begin{array}{l}\text { Durante la clase generalmente está en } \\
\text { movimiento }\end{array}$ & - & 0.79 & - \\
\hline 3. & $\begin{array}{l}\text { Interrumpe el trabajo de sus compa- } \\
\text { ñeros }\end{array}$ & - & 0.77 & - \\
\hline 4. & En clase se distrae con facilidad & 0.47 & 0.67 & - \\
\hline 15. & $\begin{array}{l}\text { En ocasiones y sin motivo aparente se } \\
\text { muestra enfadado y poco amigable }\end{array}$ & - & - & 0.75 \\
\hline 20. & $\begin{array}{l}\text { Desaprueba las sugerencias del } \\
\text { profesor }\end{array}$ & 0.40 & - & 0.62 \\
\hline 19. & Habitualmente se frustra con facilidad & - & - & 0.61 \\
\hline 17. & $\begin{array}{l}\text { Generalmente tiene dificultad para } \\
\text { controlar sus impulsos }\end{array}$ & - & 0.54 & 0.61 \\
\hline 5. & $\begin{array}{l}\text { Exige que sus demandas sean satisfe- } \\
\text { chas de inmediato }\end{array}$ & - & 0.45 & 0.52 \\
\hline
\end{tabular}

La estructura del modelo 2, con la solución completamente estandarizada y los índices de bondad de ajuste, se muestran en el cuadro 7. Se aprecia un incremento de $\chi^{2}$ de 83.30 para tres grados de libertad, lo que indica que el modelo 2 mejora significativamente al modelo 1. Si bien el modelo 2 se sostenía teóricamente, desde un punto de vista estadístico, el RMSEA seguía siendo muy elevado, por lo que se decidió indagar los índices de modificación, los cuales indicaron que el modelo mejoraba haciendo saturar el ítem 8 en el factor 1 y el ítem 17 en el factor 3.

Los índices de bondad de ajuste de este nuevo modelo (modelo 2, teniendo en cuenta los índices de modificación) fueron: $\chi^{2}=260.65$ ( $g l=162 ; p=0.00$ ); RMSEA=0.08, con un intervalo del $90 \%$ comprendido entre 0.06 y 0.11 ; GFI $=0.99$ y AGFI=0.99, además el incremento en $\chi^{2}$ fue 19.58 para dos grados de libertad, lo que supone una diferencia significativa. Estos resultados avalan empíricamente la estructura propuesta, cuya solución estandarizada se muestra en la figura 
Cuadro 6. Solución estandarizada para el modelo 1

\begin{tabular}{lccc}
\hline Items & Prob & Impuls & Desat \\
\hline 2 & 0.57 & - & - \\
6 & 0.79 & - & - \\
7 & 0.78 & - & - \\
9 & 0.63 & - & - \\
10 & 0.79 & - & - \\
11 & 0.86 & - & - \\
12 & 0.90 & - & - \\
14 & 0.84 & - & - \\
16 & 0.96 & - & - \\
18 & 0.76 & - & - \\
5 & - & 0.71 & - \\
15 & - & 0.81 & - \\
17 & - & 0.89 & - \\
19 & - & 0.71 & - \\
20 & - & 0.86 & - \\
1 & - & - & 0.72 \\
3 & - & - & 0.89 \\
4 & - & - & 0.79 \\
8 & - & - & 0.73 \\
13 & - & - & 0.85 \\
Prob & 1.00 & - & - \\
Impuls & 0.84 & 1.00 & - \\
Desat & 0.81 & 0.78 & 1.00 \\
\hline & & &
\end{tabular}

1. El ítem 8 "habitualmente no termina las actividades que inicia", además de saturar el factor de hiperactividad y desatención (factor 3), satura el factor de mala conducta (factor 1)

Cuadro 7. Solución estandarizada para el modelo 2

\begin{tabular}{lccc}
\hline Items & Prob & Impuls & Desat \\
\hline 2 & 0.57 & - & - \\
6 & 0.79 & - & - \\
7 & 0.78 & - & - \\
9 & 0.63 & - & - \\
10 & 0.79 & - & - \\
11 & 0.86 & - & - \\
12 & 0.90 & - & - \\
14 & 0.84 & - & - \\
16 & 0.92 & - & - \\
18 & 0.76 & - & - \\
5 & - & 0.71 & - \\
15 & - & 0.81 & - \\
17 & - & 0.89 & - \\
19 & - & 0.71 & - \\
20 & - & 0.86 & - \\
1 & - & - & 0.72 \\
3 & - & - & 0.89 \\
4 & - & - & 0.79 \\
8 & - & - & 0.73 \\
13 & - & - & 0.85 \\
Factor segundo orden & 0.62 & 0.19 & 0.12 \\
Prob & 1.00 & - & - \\
Impuls & 0.84 & 1.00 & - \\
Desat & 0.81 & 0.78 & 1.00 \\
\hline
\end{tabular}

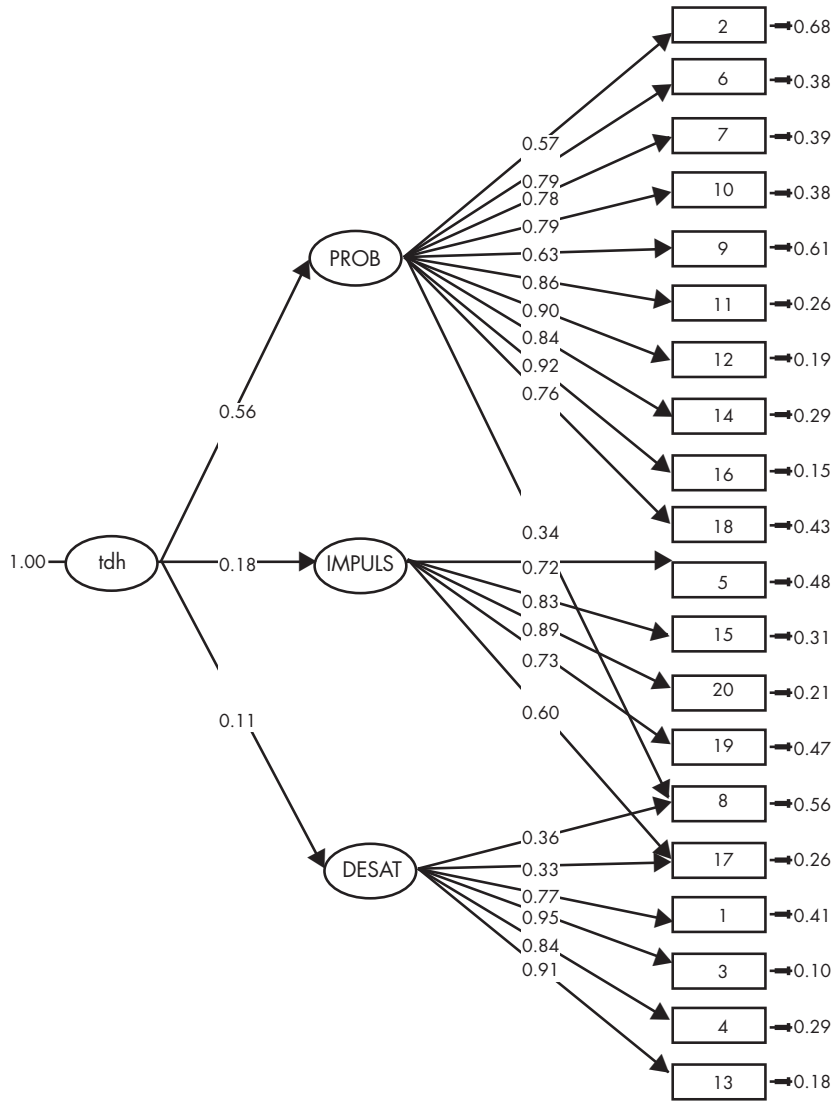

Figura 1. Modelo 2 teniendo en cuenta los índices de modificación.

parece lógico si se considera que no finalizar la tarea en la escuela es una conducta inadecuada. Por otra parte, el ítem 17 "generalmente tiene dificultad para controlar sus impulsos", además de ser un indicador de la conducta impulsiva, satura el factor de hiperactividad y desatención (factor 3) concuerda con los resultados de Farré y Narbona ${ }^{18}$ en los que hiperactividad e impulsividad componían su primer factor.

Las características psicométricas básicas de las dimensiones obtenidas en el AFC, se resumen en el cuadro 8, observándose que la fiabilidad para los factores de primer y segundo orden resultó satisfactoria. Del mismo modo, en todos los casos, la discriminación media superó el valor absoluto 0.30 , lo que indica valores adecuados.

Cuadro 8. Descriptivo en la muestra total para los factores de primer y segundo orden

\begin{tabular}{lcccc}
\hline Estadísticos & Probl & Impuls & Desat & Tdh \\
\hline Media & 18.39 & 8.27 & 10.30 & 36.96 \\
Desviación Estándar & 6.75 & 3.75 & 4.38 & 13.63 \\
Asimetría estandarizada & -0.74 & -0.23 & -0.31 & -0.58 \\
Curtosis estandarizada & 0.40 & -0.61 & -0.48 & 0.09 \\
Alfa de Cronbach & 0.92 & 0.87 & 0.89 & 0.86 \\
Discriminación media & 0.69 & 0.69 & 0.711 & 0.72 \\
Correlación corregida & $0.82^{*}$ & $0.78 *$ & $0.80^{*}$ & - \\
Factor-Total & & & & \\
\hline
\end{tabular}




\section{DISCUSIÓN Y CONCLUSIÓN}

Para tratar de dar respuesta al objetivo de la investigación, y por la necesidad de contar con instrumentos válidos y fiables para evaluar a los alumnos con TDAH, ${ }^{20,24,25}$ en esta investigación se adaptaron y se obtuvieron evidencias de validez sobre las tres dimensiones del trastorno: problemas de comportamiento, impulsividad y déficit de atención, a partir siempre de la exploración de las propiedades psicométricas de la EDAH, adaptada en una muestra de estudiantes de primaria y secundaria de Chile.

A nivel general, se obtuvieron indicadores adecuados, similares a los descritos por Farré y Narbona ${ }^{20}$ en que la media de las puntuaciones de los ítems de la escala es muy similar a la media teórica. Es decir, las propiedades psicométricas de los ítems son adecuadas. ${ }^{26,27}$ Esto permite concluir que la adaptación de la EDAH presenta características psicométricas aceptables y que, en general, muestra una adecuada consistencia interna y sus elementos poseen un adecuado poder de discriminación.

La validez de constructo, aportada por el Análisis Factorial, indicó que la estructura original propuesta por los autores no se replicaba en la población chilena, por lo cual el modelo fue sometido a un AFC. A partir del estudio de la estructura interna efectuada, es posible definir tres factores que explican los "problemas de comportamiento", la "impulsividad" y el "déficit de atención", tanto para alumnos diagnosticados con TDAH, como para aquellos que, si bien pudiesen presentar alguna sintomatología, no han sido diagnosticados y, también, en alumnos comunes. Dichos factores explicaron el $71.61 \%$ del total de la varianza, con un coeficiente Alpha de 0.948. Estas características difieren levemente de las obtenidas por Farré y Narbona, ${ }^{20}$ lo cual parece razonable si consideramos las adaptaciones realizadas y que los participantes pertenecen a contextos culturales distintos. Igualmente, las diferencias podrían deberse al sesgo de los profesores, ${ }^{28}$ puesto que si bien existe consenso en los centros respecto a la conducta y al cumplimiento de normas, la experiencia indica que el profesorado tendería a ser mayormente sancionador y poco tolerante. No obstante, la investigación logró pesquisar los problemas de conducta y de falta de atención, gracias a lo cual se encontró consistencia en las puntuaciones, pese a que distintos profesores completaron el test, en distintos escenarios educativos y con distintos alumnos. Estos resultados coinciden con otras investigaciones en que los puntajes otorgados por los profesores revelaron una buena capacidad predictiva para detectar futuros problemas de salud mental. ${ }^{29,30}$

También las diferencias podrían estar relacionadas con la adecuación semántica de la escala o con la selección de la muestra. El estudio original analizó las características de 666 alumnos (según reporte de padres y maestros), insertos en colegios de buen nivel socioeconómico; mientras que nuestra investigación contempló a 508 alumnos sin establecer dife- rencias por grupo o nivel socioeconómico. Hipotéticamente, cabría esperar que familias y profesorado se encontrasen mínimamente informados respecto al cuadro clínico estudiado, a diferencia de la información que pudiese manejar el profesorado que trabaja en escuelas chilenas públicas y subvencionadas, con alto índice de vulnerabilidad, con cursos numerosos, frecuentemente mal manejados por profesores que no poseen suficientes recursos psicopedagógicos de apoyo, y que no todos ellos tienen una capacitación específica para tratar a niños con TDAH..$^{31}$ De este modo, las expectativas, interacciones y el uso del tiempo del profesorado podrían influir sobre el rendimiento y la conducta del alumnado.

Por otra parte, el considerar dos fuentes de información para completar la escala (familias y docentes en el estudio original) si bien tiene sus ventajas, implica también asumir discrepancias en los resultados. Éstas se encuentran más bien ligadas a la valoración subjetiva que hacen los evaluadores ${ }^{18}$ y que se verían influidas por el nivel de tolerancia, de las exigencias y de las expectativas. En tal sentido, la familia habitualmente minimiza la conducta inadecuada de sus hijos, o bien su umbral de tolerancia es superior al del profesorado; razón que le lleva a asignar menores puntajes en las escalas. ${ }^{32}$ No obstante, esta circunstancia necesita ser mejor analizada mediante un estudio de validez incremental que ponga de manifiesto la contribución relativa de cada informante. ${ }^{32}$

En conclusión, la adaptación chilena de la EDAH representa los TDAH tal como la definieron sus autores. ${ }^{20}$ Además, la escala se muestra estable en dos países culturalmente diferentes. Se trata, entonces, de una escala fiable, ${ }^{33}$ con una dimensionalidad que replica el contenido teórico y unos indicadores de consistencia interna y de validez de constructo adecuados, además de una validez consecuencial que intenta responder a las exigencias sociales y orientar la toma de decisiones. ${ }^{34}$ Los efectos de la validez consecuencial movilizan hacia un cambio social de detección y mantenimiento de los tratamientos por TDAH, con grandes implicaciones en las políticas sociales, sanitarias y educativas. Esto repercute, tanto en la dinámica y organización de los centros educativos, como en la mejora de las relaciones interpersonales en el núcleo familiar.

No obstante, pese a que los resultados parecen indicar una buena bondad psicométrica de la EDAH adaptada, es necesario reseñar que los datos encontrados difícilmente podrían enmarcarse como conclusiones definitivas, puesto que, si bien las escalas tienen una función importante en la detección de los sujetos con TDAH, no existen escalas perfectas y toda confirmación de sospecha debería ser corroborada con una evaluación psicológica más detallada. ${ }^{25}$

Por otra parte, si bien los análisis realizados permiten contar con un instrumento fiable para abordar el estudio del trastorno por déficit atencional en el contexto chileno, sería de gran relevancia estudiar el comportamiento de la escala en una población más amplia y contrastar los resultados para validar el instrumento. 
Una limitación que presenta este estudio, que debería ser superada en investigaciones futuras, es que los resultados no son fácilmente generalizables a causa de lo restringido de la muestra (se seleccionó en dos provincias y no en todo el país, además de que proviene sólo de un nivel socioeconómico bajo y con un rango de edad excesivamente amplio, de 6 a 18 años, siendo que se ha demostrado que el trastorno no se manifiesta de igual forma en la infancia que en la adolescencia). ${ }^{35}$

\section{Financiamiento}

Ninguno.

\section{Conflicto de intereses}

Los autores declararon no tener conflicto de intereses.

\section{Agradecimientos}

De manera especial agradecemos a los docentes participantes y a la Unidad Infanto-Juvenil del Servicio de Psiquiatría del Hospital Guillermo Grant Benavente de Concepción-Chile su colaboración.

\section{REFERENCIAS}

1. American Psychiatric Association. Manual diagnóstico y estadístico de los trastornos mentales: DSM-IV-TR. Barcelona: Masson; 2002.

2. Organización Mundial de la Salud. Clasificación internacional de enfermedades. Décima edición. Madrid: Meditor; 1995.

3. Biederman J, Faraone S. Attention-deficit hyperactivity disorder. Lancet 2005;366:237-248.

4. Pascual I, Rafia C. Síndrome de déficit de atención con hiperactividad. Programa de formación continuada en pediatría práctica. Barcelona: Prous Science; 2001.

5. Ministerio de Salud de Chile. Atención integral de niñas/niños y adolescentes con trastorno hipercinético/trastorno de la atención (THA). Guía clínica. Santiago: Ministerio de Salud-Subsecretaría de Salud Pública; 2008.

6. Montenegro $\mathrm{H}$, Bralic $\mathrm{C}$, Edwars $\mathrm{M}$, Izquierdo $\mathrm{T}$ et al. Estandarización del inventario de problemas conductuales y destrezas sociales de $\mathrm{T}$. Achenbach en niños de seis a 11 años. Santiago de Chile: CEDEP-UNICEF; 1983.

7. Recart C, Castro O, Álvarez H, Bedregal P. Características de niños y adolescentes atendidos en un consultorio psiquiátrico del sistema privado de salud en Chile. Rev Med Chile 2002;130:295-303.

8. De la Barra F, López C, George M, Toledo V et al. Perfiles conductuales de escolares de primero básico del Área Occidente de Santiago. Revista Psiquiatría 1995;12:67-73.

9. Ministerio de Salud de Chile. Las enfermedades mentales en Chile. Magnitud y consecuencias. Santiago: Ministerio de Salud de Chile; 1999.

10. Vallés A. Alumnos con inatención, impulsividad e hiperactividad. Intervención multimodal. Madrid: EOS; 2006.

11. De la Barra F, Vicente B, Saldivia S, Melipillán R. Estudio de epidemiología psiquiátrica en niños y adolescentes en Chile. Estado actual. Rev Med Clín Las Cond 2012;23(5):521-529.

12. Vicente B, Saldivia S, De la Barra F, Melipillán R et al. Salud mental infanto-juvenil en Chile y brechas de atención sanitarias. Rev Med Chil 2012;140:447-457.
13. Lavigne R, Romero J. El TDAH. ¿Qué es?, ¿Qué lo causa?, ¿Cómo evaluarlo y tratarlo? Madrid: Pirámide; 2010.

14. López C, Belchí A, Romero A. Prevalencia/comorbilidad del TDAH. En: López S, Romero A (Coord.). TDAH y trastornos del comportamiento en la infancia y la adolescencia. Clínica, diagnóstico, evaluación y tratamiento. Madrid: Pirámide; 2013.

15. American Psychiatric Association. Diagnostic and Statistical Manual of Mental Disorders, 5th edition (DSM-5). Washington, DC: American Psychiatric Association; 2013.

16. Valdivieso A, Cornejo A, Sánchez M. Tratamiento del síndrome de déficit atencional (SDA) en niños: evaluación de la moclobemida, una alternativa no psicoestimulante. Rev Chil Neuro-psiquiatr 2000;38(suppl 1):7-14.

17. Benassini $O$. Trastornos de la atención. Origen, diagnóstico, tratamiento y enfoque psicoeducativo. Sevilla: Trillas Eduforma; 2005.

18. Barragán E, De la Peña F. Grupo de expertos nacionales para el estudio del Trastorno por Déficit de Atención e Hiperactividad. Primer consenso Latinoamericano sobre el Trastorno por Déficit de Atención e Hiperactividad. Rev Chil Psiquiatr Neurol Infanc Adolesc 2007;2:26-39.

19. Amador J, Forns M, Guàrdia J, Peró M. Estructura factorial y datos descriptivos del perfil de atención y del cuestionario TDAH para niños en edad escolar. Psicothema 2006;18(suppl 4):696-703.

20. Farré A, Narbona J. Escalas para la evaluación del trastorno por déficit de atención con hiperactividad. Madrid: TEA Ediciones; 2000.

21. Barbero I, Vila E, Holgado F. Adaptación de test en estudios comparativos interculturales. Acción Psicológica 2008;5:7-16.

22. Kaiser $\mathbf{H}$. The Varimax criterior for analytic rotation in factor analysis. Psychometrika 1958;23:187-200.

23. Farré A, Narbona J. Índice de hiperquinesia y rendimiento escolar: validación del cuestionario de Conners en nuestro medio. Acta Pediátrica Esp 1998;47:103-109.

24. Conners C. Conner's Parent Rating Scale-Revised Manual. Nueva York: Multi-Health Systems; 1997.

25. Parellada M. TDAH Trastorno por déficit de atención e hiperactividad. De la infancia a la edad adulta. Madrid: Alianza; 2009.

26. Carretero-Dios H, Pérez C. Normas para el desarrollo revisión de estudios instrumentales. International J Clinical Health Psychology 2005;5:521-551.

27. Muñiz J. Medio siglo de teoría de respuesta a los ítems. Anuario Psicología 1992;52:41-66.

28. Ablow J, Measelle J, Kraemer $\mathrm{H}$ et al. The MacArthur three-city outcome study: Evaluating multi-informant measures of young children's symptomatology. J Am Acad Child Adolesc Psychiatry 1999;38:1580-1590.

29. Achenbach T, Howell C, McConaughy S, Stanger C. Six year predictors of problems in a national sample of children \& youth: II. Signs of disturbance. J Am Acad Child Adolesc Psychiatry 1995;34:488-498.

30. Kellam S, Ling X, Merisca R, Brown C et al. The effect of the level of aggression in the first grade classroom on the course and malleability of aggressive behavior into middle school. Development Psychopathology 1998;10:165-185.

31. Díaz M, Trujillo M. Eficacia de un protocolo de evaluación multi-metodológico para el diagnóstico del trastorno por déficit de atención con hiperactividad. Rev Chil Psiquiatr Neurol Infanc Adolesc 2008;8(suppl 1):1-11.

32. Carrasco M, Holgado F, Del Barrio M, Barbero I. Validez incremental: un estudio aplicado con diversas fuentes informantes y medidas. Acción Psicológica 2008;5:65-76.

33. Vicente B, Navas L, Belmar M, Holgado F. Análisis de la escala para la evaluación del trastorno por déficit de atención con hiperactividad en población escolar chilena. Rev Med Chile 2010;138:1497-1504.

34. Padilla J, Gómez J, Hidalgo M, Muñiz J. La evaluación de las consecuencias del uso de los test en la teoría de la validez. Psicothema 2007;18(suppl 2):307-312.

35. American Psychiatric Association. Manual diagnóstico y estadístico de los trastornos mentales. DSM-5. Madrid: Editorial Médica Panamericana; 2014. 